\title{
Application of Extended Jiles-Atherton Model for Modelling the Influence of Stresses on Magnetic Characteristics of the Construction Steel
}

\author{
D. JACKIEWICZ ${ }^{a, *}$, R. SzEWCZYK ${ }^{b}, \mathrm{~J}$. SAlACH ${ }^{b}$, A. BieńKOWSKI ${ }^{b}$ \\ ${ }^{a}$ Industrial Research Institute for Automation and Measurements,Al. Jerozolimskie 202, 02-486 Warsaw, Poland \\ ${ }^{b}$ Institute of Metrology and Biomedical Engineering, Warsaw Universityof Technology, Poland
}

Paper presents the possibility of application of the Jiles-Atherton extended model to describe the magnetic characteristics of construction steel $\mathrm{C} 45$ under the influence of tensile stresses. Experiment was performed on the frame-shaped samples. Then, the influence of stresses on hysteresis loops was modelled with the Jiles-Atherton extended model. The obtained results of the modelling are consistent with results of the experimental measurements. The results of modelling create new possibilities of explanation of the physical phenomena connected with magnetisation of the magnetic materials under stresses, which is esential for the assessment of the state of the construction steel during its exploitation in industrial conditions.

DOI: 10.12693/APhysPolA.126.392

PACS: 75.60.Ej, 75.60.-d, 81.70.-q

\section{Introduction}

Magnetoelastic effect is connected with changes of magnetization of the material under the influence of mechanical stresses [1]. This effect was widely investigated in soft magnetic alloys, both amorphous [2] and nanocrystaline [3] due to the possibility of development of robust force sensors [4]. Moreover, magnetoelastic effect is significant from the point of view of development of new methods of non-destructive testing of the ferromagnetic materials, mainly steels [5].

However, for effective stress assessment in the material, a quantitative model of stress dependence of the magnetic hysteresis loops is required. One of the most suitable models for such purposes is Jiles-Atherton model [6-11] with extension considering changing of pinning site properties during the magnetisation process [12].

\section{Methods of experiments and modelling}

Experimental data was acquired with the use of a frame-shaped sample made of the $\mathrm{C} 45$ steel. The sample was subjected to tensile stresses with the use of a material testing machine [13]. During the measurements, the applied stress was increased up to $500 \mathrm{MPa}$, which is near the point of rupture of the sample.

Then, the influence of stresses on the hysteresis loops was modelled with the Jiles-Atherton extended model. Parameters of the model were determined during the optimisation process with the use of evolutionary strategy $(\mu+\lambda)$ together with gradient optimisation [12]. The

*corresponding author; e-mail: d.jackiewicz@mchtr.pw.edu.pl modelling was carried-out for each value of stresses influencing magnetic characteristics of $\mathrm{C} 45$ steel.

However, parameters were determined simultaneously for three $B(H)$ hysteresis loop. As a result, parameters of extended Jiles-Atheton model describe magnetic properties of this steel in wide range of amplitude of the magnetizing field.

\section{Results and conclusions}

Figure 1 presents the experimental results of measurements of stress dependence of magnetic characteristics of frame-shaped samples made of C45 steel. Stress dependence of the shape of hysteresis $B(H)$ loops may be observed in Figs 1a and 1b. This dependence is well described by extended Jiles-Atherton model, which is confirmed by the fact, that $R^{2}$ Pearson coefficient exceeds $95 \%$ for all values of amplitude of magnetizing field. Figure 1c presents magnetoelastic $B(\sigma)_{H m}$ dependences, obtained for three given values of amplitude of magnetizing field $\mathrm{H}_{m}: 400 \mathrm{~A} / \mathrm{m}, 740 \mathrm{~A} / \mathrm{m}$ and $2480 \mathrm{~A} / \mathrm{m}$. It should be indicated, that shape of the $B(\sigma)_{H m}$ dependences is very well featured by the results of modelling. Moreover, maximum on the $B(\sigma)_{H m}$ dependences (Villari point) may be observed in both experimental results as well as in the results of modelling.

Figure 2 presents the dependence of selected, extended Jiles-Atherton model's parameters. It was observed, that parameters $a$ describing domain wall density, as well as parameter $\alpha$ connected with inter domain coupling, exhibit extremes for values of stresses $\sigma$ near the Villari point and the yield point. However, magnetisation reversibility $c$ decreases for higher values of compressive stresses $\sigma$, as well as anisotropy energy density increases up to Villari point and yield point. These phenomena are in good agreement with quantitative description of 

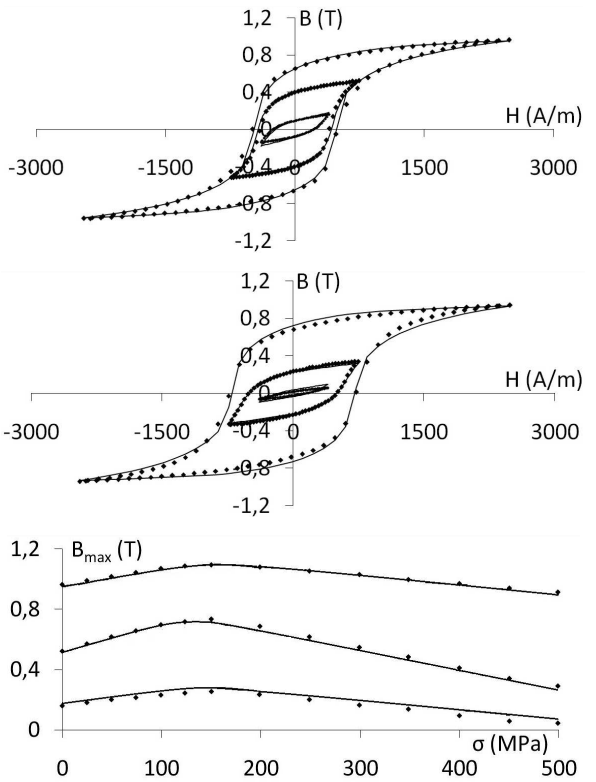

Fig. 1. Experimental results $(\checkmark)$ and the results of modelling (-): a) $\mathrm{B}(\mathrm{H})$ hysteresis loop without stresses, b) $\mathrm{B}(\mathrm{H})$ hysteresis loop under stresses $\sigma=500 \mathrm{MPa}, \mathrm{c}$ ) magnetoelastic $B(\sigma)_{H}$ characteristics.
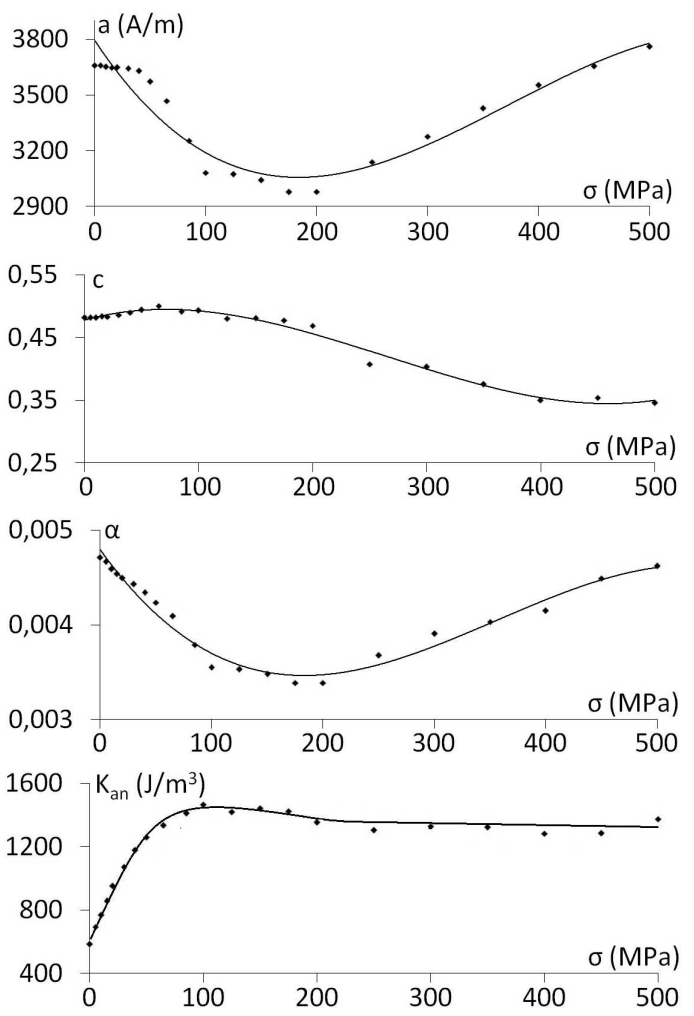

Fig. 2. Stress dependence of extended Jiles-Atherton model parameters: a) $a$ - describing domain walls density, b) magnetization reversibility $c$, c) $\alpha$ - describing inter domain coupling, d) anisotropy energy density $K_{a n}(-$-trend line). magnetization process in crystalline materials. Saturation magnetisation $M_{s}$ is constant. Due to the fact that it depends only on the physical properties of the material, such as its chemical composition, stresses do not influence the saturation magnetisation.

Results presented in this paper are filling the gap connected with modelling of stress dependence of magnetic hysteresis loop of C45 construction steel. As a conclusion presented results are step towards practical use of quantitative magnetic hysteresis loop modelling in nondestructive testing of construction elements.

\section{Acknowledgments}

This work was partially supported by The National Centre of Research and Development (Poland) within grant PBS1/B4/6/2012. Calculations for the modelling were made in the Interdisciplinary Centre for Mathematical and Computational Modelling of Warsaw University (G36-10).

\section{References}

[1] M.J. Sablik, D.C. Jiles, IEEE Trans. Magn. 29, 2113 (1993).

[2] Z. Stoklosa, J. Rasek, P. Kwapulinski, G. Badura, G. Haneczoka, L. Pajak, J. Lelatko, A. KolanoBurian, J. Alloys Compd. 509, 9050 (2011).

[3] T. Kulik, J. Ferenc, A. Kolano-Burian, X. Liang, M. Kowalczyk, J. Alloy. Compd. 434-435, 623 (2007).

[4] A. Bieńkowski, R. Szewczyk, Sens. Actuators A 113 , 270 (2004).

[5] L. Piotrowski, B. Augustyniak, M. Chmielewski, J. Labanowski, M. Lech-Grega, DT \& E International 47, 157 (2012).

[6] D.C. Jiles, J. Magn. Magn. Mater. 61, 48 (1986).

[7] D.L. Atherton, D.C. Jiles, NDT International 19, 15 (1986).

[8] M. Hamimid, S.M. Mimoune, M. Feliachi, Phys. B 406, 2755 (2011).

[9] K. Chwastek, J. Phys. D 43, 015005 (2010).

[10] J. Li, M. Xu, J. Appl. Phys. 110, 063918 (2011).

[11] R. Szewczyk, Acta Phys. Pol. A 113, 67 (2008).

[12] R. Szewczyk, J. Salach, A. Bieńkowski, P. Frydrych, A. Kolano-Burian, IEEE Trans. Magn. 48, 1389 (2012).

[13] D. Jackiewicz, R. Szewczyk, J. Salach, Theoretical and Applied Informatics 25, 17 (2013). 